\title{
(6) OPEN ACCESS \\ Temporal lobe epilepsy and affective disorders: the role of the subgenual anterior cingulate cortex
}

\author{
J Stretton, ${ }^{1,2}$ R A Pope, ${ }^{1,3}$ G P Winston, ${ }^{1}$ M K Sidhu, ${ }^{1}$ M Symms, ${ }^{1}$ J S Duncan, ${ }^{1}$ \\ M Koepp, ${ }^{1}$ P J Thompson, ${ }^{1}$ J Foong ${ }^{1}$
}

${ }^{1}$ Epilepsy Society MRI Unit, Department of Clinical and Experimental Epilepsy, Institute of Neurology, University College London, London, UK ${ }^{2}$ MRC Cognition and Brain Science Unit, Cambridge, UK ${ }^{3}$ Clinical Psychopharmacology Unit, University College London, London, UK

\section{Correspondence to} Rebecca A Pope, Clinical Psychopharmacology Unit, University College London, Gower Street, London WC1E 6BT, UK; r.pope@ucl.ac.uk

JS and RAP are joint first authors.

Received 10 October 2013 Revised 6 April 2014 Accepted 23 April 2014 Published Online First 29 May 2014

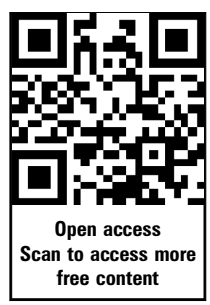

CrossMark

To cite: Stretton J Pope RA, Winston GP, et al. J Neurol Neurosurg Psychiatry 2015;86: 144-151.

\section{ABSTRACT}

Objective Reduced deactivation within the default mode network (DMN) is common in individuals with primary affective disorders relative to healthy volunteers (HVs). It is unknown whether similar network abnormalities are present in temporal lobe epilepsy (TLE) patients with a history of affective psychopathology. Methods 17 TLE patients with a lifetime affective diagnosis, 31 TLE patients with no formal psychiatric history and $30 \mathrm{HVs}$ were included. We used a visuospatial 'n-back' paradigm to compare working memory (WM) network activation between these groups. Post hoc analyses included voxel-based morphometry and diffusion tensor imaging. The Beck Depression InventoryFast Screen and Beck Anxiety Inventory were completed on the day of scanning.

Findings Each group activated the fronto-parietal WM networks and deactivated the typical DMN in response to increasing task demands. Group comparison revealed that TLE patients with lifetime affective morbidity showed significantly greater deactivation in subgenual anterior cingulate cortex (SACC) than either the TLE-only or the HVs $(p<0.001)$. This effect persisted after covarying for current psychotropic medication and severity of current depressive/anxiety symptoms (all $p<0.001$ ). Correlational analysis revealed that this finding was not driven by differences in task performance. There were no significant differences in grey matter volume or structural connectivity between the TLE groups.

Conclusions Our results provide novel evidence suggesting that affective psychopathology in TLE has a neurobiological correlate, and in this context the SACC performs differently compared with network activity in primary affective disorders.

\section{INTRODUCTION}

Temporal lobe epilepsy (TLE) is associated with marked psychiatric comorbidity. ${ }^{1} 2$ Affective disorders (depression/anxiety) are the most common interictal psychopathologies, ${ }^{3}$ leading to a decreased quality of life ${ }^{4}$ and an increased risk of suicide. ${ }^{2}$

Historically, affective disorders in epilepsy have been conceptualised as a complication of the underlying seizure disorder. ${ }^{6}$ Recent epidemiological studies however suggest a more complex relationship, as a history of major depression ${ }^{7-11}$ or anxiety disorders ${ }^{1011}$ is associated with an increased risk of developing epilepsy. Moreover, a lifetime history of affective psychopathology has been associated with a poorer response to antiepileptic medication ${ }^{12}$ and epilepsy surgery. ${ }^{13-16}$
The primary affective disorder literature suggests that dysfunction within an extended network including the medial prefrontal cortex (mPFC) and anatomically-related limbic, striatal, thalamic and basal forebrain structures is pathogenic in the onset and maintenance of affective morbidity. ${ }^{17} 18$ In parallel, evidence from structural ${ }^{19-27}$ and functional neuroimaging ${ }^{28-37}$ suggests the same structures may be involved in the development and maintenance of affective symptoms in TLE.

More recently, functional imaging studies have focused on the default mode network (DMN), ${ }^{38} 39$ a resting-state network that putatively mediates task-independent, self-referential cognition and interactive modulation between intrinsic activities and external tasks. ${ }^{40}$ DMN dysfunction has been implicated in psychiatric diagnoses. ${ }^{41}{ }^{42}$ Studies of primary unipolar and bipolar depression have reported reduced deactivation of the DMN during self-referential, ${ }^{43}$ emotional processing ${ }^{44} 45$ and verbal working memory (WM) tasks ${ }^{46}$ with altered patterns of functional connectivity at rest relative to healthy brains. ${ }^{47} 48$ DMN dysfunction has also been demonstrated in anxiety disorders $\left(\mathrm{see}^{49}\right.$ for review).

Studies of the DMN in TLE have indicated reduced functional connectivity ${ }^{50} 51$ and altered task-related deactivations compared with healthy controls. $^{52} 53$ Emerging evidence suggests that DMN connectivity may be further complicated by comorbid psychopathology. ${ }^{54-56}$ Chen et $a l^{54}$ demonstrated that in depressed mTLE patients, functional connectivity among the anterior prefrontal cortex, limbic system and temporal lobe is significantly reduced compared with non-depressed TLE patients and healthy controls. However, it is still unknown whether comorbid affective morbidity in TLE modulates the behaviour of this neural network.

WM tasks are normally associated with deactivation of the DMN. ${ }^{57}$ The aim of this study was to examine DMN function and structure in patients with TLE and a history of comorbid affective disorders. Specifically, we hypothesised that patients with a lifetime affective diagnosis would demonstrate reduced deactivation of the $\mathrm{DMN}$ during a WM task, similar to patients with primary affective disorders, ${ }^{40-42}$ and this would be associated with poorer performance.

\section{MATERIALS AND METHODS Subjects}

A total of 48 right-handed patients with medically refractory TLE undergoing presurgical evaluation 
Table 1 Demographic and clinical characteristics for patients and healthy volunteers (HVs)

\begin{tabular}{|c|c|c|c|c|}
\hline & PHx $(n=17)$ & $n P H x(n=31)$ & HV $(n=30)$ & p Value \\
\hline Age, years* & $40(17-53)$ & $38(19-55)$ & $37(19-64)$ & 0.74 \\
\hline Female & $14(82 \%)$ & $17(55 \%)$ & $18(60 \%)$ & 0.20 \\
\hline Lesion laterality (R/L) & $7 / 6(41 / 35 \%)$ & $11 / 16(35 / 52 \%)$ & NA & 0.44 \\
\hline Epilepsy onset, yearst & $14(7.5-25)$ & $17(11-22)$ & NA & 0.94 \\
\hline Epilepsy duration, yearst & $21(10-30)$ & $19(10-30)$ & NA & 0.70 \\
\hline MRI negative & $4(23 \%)$ & $4(13 \%)$ & NA & 0.43 \\
\hline HS & $7(41 \%)$ & $20(64 \%)$ & NA & 0.12 \\
\hline Cavernomas & $2(12 \%)$ & $2(6.5 \%)$ & NA & 0.61 \\
\hline DNET & $2(12 \%)$ & $5(16 \%)$ & NA & 1.00 \\
\hline FCD & $0(0 \%)$ & $2(6.5 \%)$ & NA & 0.53 \\
\hline Other $¥$ & $2(12 \%)$ & $3(10 \%)$ & NA & 1.00 \\
\hline Dual pathology & $0(0 \%)$ & $5(16 \%)$ & NA & 0.15 \\
\hline Number of AEDs* & $3(1-4)$ & $2(1-4)$ & NA & 0.28 \\
\hline Premorbid IQ§ & $99.5(17)$ & $97.4(12.3)$ & $111(10.6) \rrbracket$ & \\
\hline BDI-FS* & $4(0-12)$ & $2(0-9)$ & $0.5(0-5) \rrbracket$ & \\
\hline $\mathrm{BAI}^{*}$ & $9(2-33)$ & $6(0-28)$ & $2.5(0-12) 9$ & \\
\hline \multicolumn{5}{|l|}{ Lifetime psychiatric history: } \\
\hline Unipolar depression & $12(70 \%)$ & NA & NA & \\
\hline Anxiety disorder & $3(18 \%)$ & NA & NA & \\
\hline Depression and anxiety & $2(12 \%)$ & NA & NA & \\
\hline \multicolumn{5}{|l|}{$\begin{array}{l}\text { *Median (range). } \\
\text { †Median (IQR). } \\
\text { †Abnormal gyral folding/cal } \\
\text { §Mean (SD). }\end{array}$} \\
\hline
\end{tabular}

at the National Hospital for Neurology and Neurosurgery participated in this study between 2009 and 2012. Seventeen patients had at least one lifetime episode of an affective disorder (psychiatric history (PHx) group). Thirty-one patients had no history of psychiatric disorder (none-psychiatric history (nPHx) group). We also recruited 30 native English speaking healthy volunteers (HVs) without any history of neurological or psychiatric disease. Clinical and demographic data are detailed in table 1.

All patients had a structural MRI at 3 Tesla (3 T). Video-scalp EEG had confirmed seizure onset in the medial temporal lobe. All patients were taking antiepileptic medication, were native English speakers and had undergone a neuropsychological and neuropsychiatric evaluation as part of presurgical investigations. Seven of the 17 patients with a psychiatric history had a current psychiatric diagnosis (six unipolar depression; one anxiety disorder); 5 (29\%) were prescribed antidepressant or anxiolytic medication.

The study was approved by the National Hospital for Neurology and Neurosurgery and the Institute of Neurology Joint Research Ethics Committee, and written informed consent was obtained from all subjects.

\section{Neuropsychiatric evaluation and analysis}

Neuropsychiatric diagnosis: lifetime and current

All patients were assessed by a neuropsychiatrist (JF) via a clinical interview. The diagnoses of mood and anxiety disorders fulfilled criteria according to the Diagnostic and Statistical Manual of Mental Disorders (DSM-IV-TR). Patients with affective symptoms related to antiepileptic (antiepileptic drug (AED)) therapy or the peri-ictal period (symptoms temporally related to seizure activity) were excluded from the study. We also excluded patients with a history of postictal or interictal psychosis.
Beck Depression and Anxiety Inventories (BDI-FS and BAI)

All subjects completed the Beck Depression Inventory-Fast Screen $\left(\mathrm{BDI}-\mathrm{FS}^{58}\right.$ ) and Beck Anxiety Inventory $\left(\mathrm{BAI}^{59}\right)$. For both inventories, recommended severity ranges were employed (BDI-FS: 0-3 minimal; 4-6 mild; 7-9 moderate; 10-21 severe. BAI: 0-7 minimal; 8-15 mild; 16-25 moderate; 26-63 severe).

\section{MR data acquisition}

MRI studies were performed on a $3 \mathrm{~T}$ General Electric Excite HDx scanner. Standard imaging gradients with a maximum strength of $40 \mathrm{~m} \mathrm{Tm}^{-1}$ and slew rate $150 \mathrm{Tm}^{-1} \mathrm{~s}^{-1}$ were used. All data were acquired using an eight-channel array head coil for reception and the body coil for transmission. Standard clinical sequences were performed and included a coronal T1-weighted volumetric acquisition with 170 contiguous $1.1 \mathrm{~mm}$ thick slices (matrix $256 \times 256$, inplane resolution $0.9375 \times 0.9375 \mathrm{~mm}$ ) used for voxel-based morphometry (VBM) analysis.

For the fMRI task, gradient-echo planar T2*-weighted images were acquired, providing blood oxygenation level-dependent (BOLD) contrast. A total of 272 volumes were acquired over 1 run lasting $11 \mathrm{~min} 20 \mathrm{~s}$. Each volume comprised 50 oblique axial $2.4 \mathrm{~mm}$ slices (with $0.1 \mathrm{~mm}$ gap) covering the whole brain, with a $24 \mathrm{~cm}$ field of view, SENSE factor $2,64 \times 64$ matrix and an inplane resolution of $3.75 \times 3.75 \mathrm{~mm}^{3}$. Echo time was $25 \mathrm{~ms}$ and repetition time was $2.5 \mathrm{~s}$.

\section{Diffusion tensor imaging acquisition}

Data were acquired using a cardiac-triggered single-shot spin-echo planar imaging sequence with echo time $=73 \mathrm{~ms}$. Sets of 60 contiguous $2.4 \mathrm{~mm}$ thick axial slices were obtained covering the whole brain, with diffusion sensitising gradients applied in each of 52 non-collinear directions (b value of $1200 \mathrm{~mm}^{2} \mathrm{~s}^{-1}$ 
$(\delta=21 \mathrm{~ms}, \Delta=29 \mathrm{~ms})$ using full gradient strength of $40 \mathrm{mT} \mathrm{m}^{-1}$ ) along with six non-diffusion-weighted scans. Gradient directions were calculated and ordered as described elsewhere. ${ }^{60}$ Field of view was $24 \mathrm{~cm}$, with matrix size of $96 \times 96$, zero filled to $128 \times 128$ during reconstruction, giving a reconstructed voxel size of $1.875 \times 1.875 \times 2.4 \mathrm{~mm}$. The first four scans were discarded to ensure magnetisation equilibrium.

\section{'Dot-back' fMRI paradigm and data analysis}

A modified version of the 'n-back' task was used. ${ }^{53}{ }^{61}$ Subjects were required to monitor the locations of dots (presentation time $440 \mathrm{~ms}$; inter-stimulus interval $1500 \mathrm{~ms}$ ) at a given delay from the original occurrence. There were three $30 \mathrm{~s}$ active conditions in total (0-, 1- and 2-back) presented to subjects five times in pseudorandom order, controlling for any order effect. Each block contained a maximum of 15 correct responses. Each active condition started with a $15 \mathrm{~s}$ resting baseline, with a further $15 \mathrm{~s}$ rest between active blocks during which the word 'rest' and all four dots were presented on screen, with the instruction to watch the screen. Subjects were required to move the joystick corresponding to the correct location of the current (0-back) or previously presented stimulus (1-back=previous presentation; 2 -back $=$ previous presentation but one; chance performance $=25 \%$ ). Percentages of correct 0,1 and 2 dot-back trials were used in subsequent analysis as a measure of performance.

\section{DATA ANALYSIS}

Imaging data were analysed with Statistical Parametric Mapping (SPM8) (http://www.fil.ion.ucl.ac.uk) using a two-level random-effects analysis. The imaging time series of each subject was realigned using the mean image as reference, spatially normalised into standard anatomical space (scanner-specific template) using the high-resolution whole brain-echo planar image and smoothed with a Gaussian kernel of $8 \mathrm{~mm}$ FWHM.

At the first level, trial-specific responses were modelled for each subject by convolving a $\delta$ function that indicated each block onset with the canonical haemodynamic response function (HRF) to create regressors of interest, one regressor for each block ('0-back', '1-back' and '2-back'). Each subject's movement parameters were included as confounds, and parameter estimates pertaining to the height of the HRF for each regressor of interest were calculated for each voxel. A single contrast image for the main effect of the DMN, modelling areas of increasingly negative blood-oxygen level-dependent (BOLD) signal change in response to increasing task demand, ${ }^{62}$ was created for each subject. This contrast image was then used for the second-level analysis.

At the second level of the random-effects analysis, the subjects were divided into three groups: $\mathrm{HV}, \mathrm{nPHx}$ and $\mathrm{PHx}$. Analysis of covariance (ANCOVA) was performed with group as a factor to examine the main effects of the contrast and to highlight regions demonstrating more or less deactivation in one group compared with another. Gender, BDI-FS and BAI were included as regressors of no interest in all analyses. We report all activations at a threshold of $\mathrm{p}<0.001$, uncorrected for multiple comparisons. Post hoc analyses were performed to explore possible confounding factors associated with a significant main effect. No exams were discarded due to movement.

\section{Post hoc imaging analyses}

Voxel-based morphometry

The T1-weighted images were segmented into grey matter, white matter and cerebrospinal fluid using VBM8. Grey matter segmentations were normalised to MNI space and smoothed with a Gaussian kernel of $8 \mathrm{~mm}$ FWHM to produce maps of grey matter volume (GMV). An ANCOVA was performed to determine group differences in GMV.

\section{Fractional anisotropy and structural connectivity analysis}

Diffusion tensor imaging data were preprocessed to remove motion artefact and eddy current distortion using the FSL eddycorrect routine. Maps of fractional anisotropy (FA) as an indicator of structural integrity were obtained using the FMRIB Diffusion Toolbox. They were non-linearly registered to the FMRIB58 template using FLIRT and FNIRT ${ }^{63}$ and smoothed with a Gaussian kernel of $8 \mathrm{~mm}$ FWHM. A whole brain voxelwise ANCOVA of the FA maps between groups was undertaken in SPM8 with gender, BDI-FS and BAI scores included as regressors of no interest.

Tractography was performed in each subject to investigate the connectivity from the region identified by fMRI. A $6 \mathrm{~mm}$ sphere was defined in the white matter of the anterior corpus callosum underlying the activation maxima of the general linear model ANCOVA in the normalised space of the FMRIB58 template. This was transformed into each individual's native space using the inverse of transform calculated using FNIRT. Probabilistic tractography was then performed in native space from this seed using the FSL Diffusion Toolkit. ${ }^{64}$ The connectivity maps generated for each subject were scaled by the number of streamlines initiated and normalised to the FMRIB58 template using the previously calculated transformation and smoothed with a Gaussian kernel of $8 \mathrm{~mm}$ FWHM. A whole brain voxelwise comparison of the structural connectivity maps between groups was undertaken in SPM8 with gender, BDI-FS and BAI scores included as regressors of no interest.

\section{RESULTS}

\section{Beck Depression and Anxiety Inventories}

ANOVA revealed both TLE groups had significantly higher BDI-FS and BAI scores than controls on the day of scanning $(p<0.01)$. There were no significant differences between patient groups on these measures (see table 1).

\section{Inscanner performance}

ANOVA revealed a main effect of group on the 0 -back ( $F$ $(2,75)=4.84, \mathrm{p}<0.05), 1$-back $(\mathrm{F}(2,75)=7.18, \mathrm{p}<0.05)$ and 2-back conditions (F $(2,75)=3.52, \mathrm{p}<0.05)$. Post hoc Bonferroni analysis showed the $\mathrm{nPHx}$ group performed significantly less well than HVs during the 0 dot-back condition $(p=0.008)$. The PHx group also performed less well than HVs during the 0-back condition; however, this was not statistically significant. There was no significant difference between the patient groups during the 0 -back condition. In the 1 dot-back condition, the $\mathrm{PHx}$ group performed significantly worse than the HVs $(p<0.001)$ and the nPHx group $(p=0.049)$. In the 2 dot-back condition, the PHx group performed significantly less well than HVs $(p=.029)$; however, their performance was not significantly different to the $n \mathrm{PHx}$ group $(\mathrm{p}=0.70)$. There was no significant difference between the $\mathrm{HV}$ and $\mathrm{nPHx}$ groups in either 1 or 2 dot-back conditions (see figure 1 ).

\section{fMRI default mode network}

Each group progressively deactivated the typical DMN consisting of the precuneus, medial frontal gyrus, anterior cingulate cortex (ACC), superior temporal gyri and hippocampi in response to increasing task demands (figure 2). ANCOVA revealed the $\mathrm{PHx}$ group additionally deactivated the subgenual ACC ( $\mathrm{sACC})(\mathrm{x}=2, \mathrm{y}=28, \mathrm{z}=-2)$ significantly more than the $\mathrm{HV}$ and the nPHx groups (figure 3 ). 
Figure 1 Group results across WM dot-back conditions $\left({ }^{*}=p<.05\right)$.

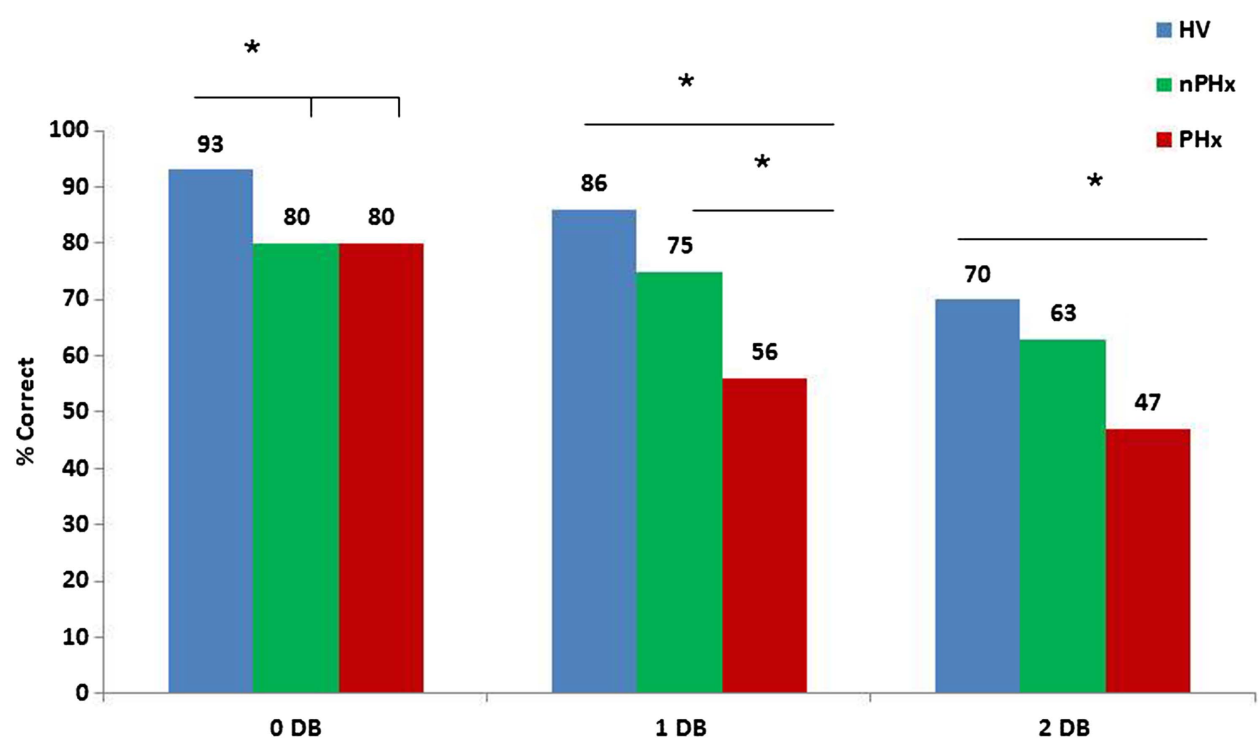

\section{Post hoc fMRI analyses}

To determine whether the result for the $\mathrm{PHx}$ group may be influenced by current affective diagnosis, the data were reanalysed in a separate ANCOVA dividing the PHx group into lifetime $(n=10)$ versus current $(n=7)$ psychiatric diagnosis. There was no significant difference in the deactivation between these groups. Two further ANCOVAs were performed excluding those patients in the PHx group with scores falling in the moderate to severe range on the BDI-FS $(n=3$ removed) and BAI $(n=4$ removed). Both analyses revealed the same effect with significantly greater sACC deactivation $(x=2, y=28, z=-2)$ in the $\mathrm{PHx}$ group compared with both the HV and nPHx groups.

A further ANCOVA was performed to explore the potential role of current psychiatric medication. The five patients taking antidepressant and anxiolytic medication were removed from the analysis. The same effect was observed in that the PHx group deactivated the same sACC cluster $(x=2, y=30, z=-4)$ significantly more than $\mathrm{HV}$ and the $\mathrm{nPHx}$ groups.

Finally, to ensure the result was not driven by differences in task performance, correlational analyses were performed between the inscanner performance on each condition and the sACC BOLD parameter estimates for each subject. There was no significant linear correlation between performance and sACC signal in the 0,1 or 2 dot-back conditions. There were no significant correlations between BDI-FS and BAI scores and sACC signal. In addition, as a manipulation check, all three groups progressively activated the expected fronto-parietal WM networks as a function of increasing WM demand.

\section{Voxel-based morphometry}

A whole brain VBM analysis was conducted to examine whether the main effect of group on DMN deactivation in the sACC was associated with structural differences in this region. There were no significant differences in GMV between the groups (all $\mathrm{p}$ $>0.05)$.

\section{FA maps}

To explore white matter integrity a whole brain voxelwise ANCOVA was performed using the FA maps generated at the individual level. There were no significant group differences in FA between the PHx group and HVs, or the nPHx group.

\section{Structural connectivity}

Seeding from the anterior corpus callosum, each group showed structural connectivity to the forceps minor bilaterally. ANCOVA revealed no significant group differences in the structural connectivity of this region.

\section{DISCUSSION}

We report task-related DMN deactivation in patients with TLE and a diagnosed history of comorbid affective disorders. Contrary to our prediction, TLE patients with and without a history of affective disorders and HV demonstrated a progressive deactivation of the DMN in response to increasing WM task demands. Furthermore, the $\mathrm{PHx}$ group showed additional deactivation in the sACC, a mesolimbic structure implicated in the pathophysiology of primary depression. ${ }^{65-67}$
Figure 2 Group results for progressive deactivation of the typical default mode network in response to increasing task demand ( $p<.001$, unc); controlling for both gender and current mood (BDI-FS and BAl scores). The superior temporal gyrus (STG) is not visualised owing to slice selection to show maximum default mode network deactivation.

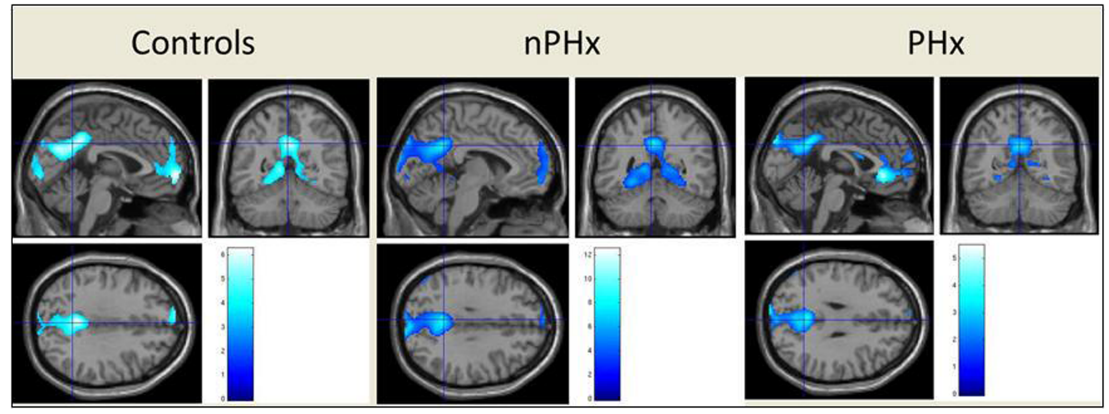



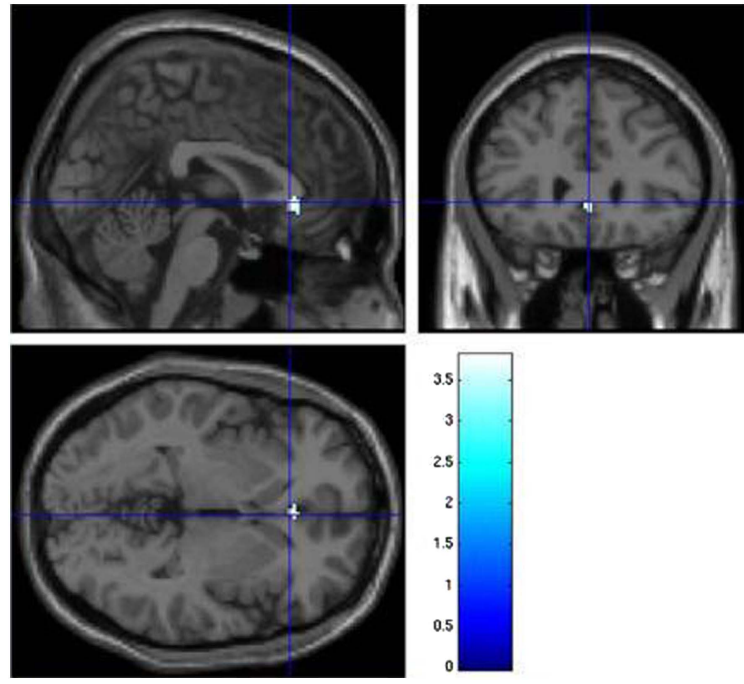

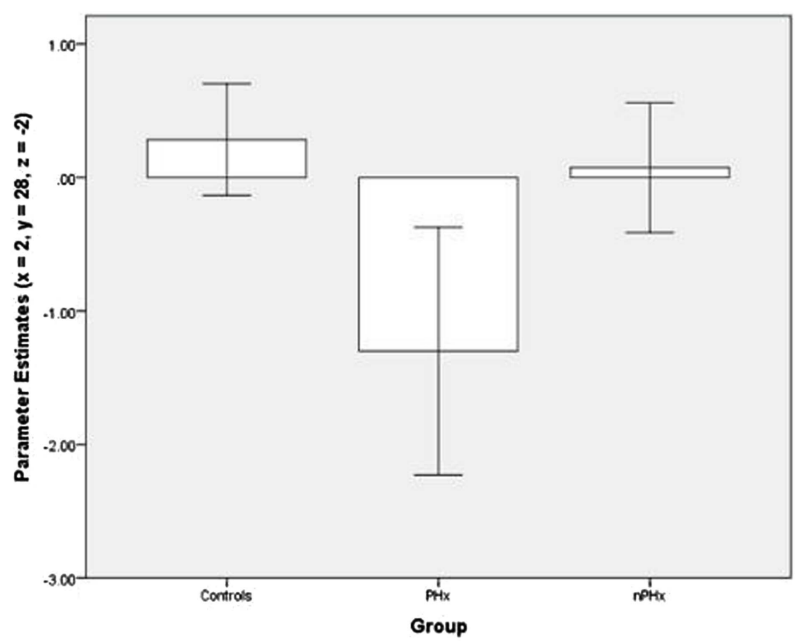

Figure 3 Additional deactivation of the $\operatorname{sACC}(x=2, y=28, z=-2)$ in the PHx group only $(p<.001$, unc); controlling for both gender and current mood (BDI-FS and BAl scores).

Increased deactivation within the DMN in the PHx group contrasts with the growing literature demonstrating that the DMN is less deactivated in affective disorders during selfreferential, ${ }^{23}$ emotional processing ${ }^{24}{ }^{25}$ and verbal WM tasks. ${ }^{26}$ Available evidence does indicate that DMN activity is significantly attenuated in TLE with seizures that impair awareness (see ${ }^{68}$ for review).

EEG-fMRI studies have consistently demonstrated interictal epileptic discharges (IED)-related deactivations in the anterior $^{769}$ and posterior ${ }^{69-71}$ nodes of the DMN in TLE patients during rest. Laufs et $a l^{69}$ suggested DMN deactivation in TLE is facilitated by the propagation of interictal epileptic activity to functionally interconnected default brain regions, resulting in secondary inhibition of non-seizing cortical regions via subcortical structures. We observed task-related deactivation in the $\mathrm{PHx}$ group that was significantly greater than that which occurred in the $\mathrm{nPHx}$, and in a component of the 'affective network' (sACC), ${ }^{40}$ supporting an additional, more specific, epilepsy-affective interaction. However, it is also possible that the rate of IEDs was dissimilar between the patient groups, particularly given the evidence that affective morbidity has been reported as predictive of seizure recurrence in newly treated patients $^{72}$ and has been associated with a poorer response to antiepileptic medication $^{12}$ and epilepsy surgery. ${ }^{16}$ 73-75 As we did not collect simultaneous acquisition of EEG-fMRI data, we are unable to examine whether interictal epileptiform activity mediated the relation between sACC deactivation and/or cognitive performance in the $\mathrm{PHx}$ group. ${ }^{76}$

Our post hoc analyses indicated that the main effect of group on DMN deactivation of the sACC was not related to volumetric or structural connectivity differences of this region, but represents an isolated functional abnormality. Further studies using resting-state EEG-fMRI methodology are needed to explore this interactional physiological interpretation.

Functional neuroimaging techniques suggest that the ACC is functionally and structurally heterogeneous. ${ }^{77}$ A meta-analyses of task-based studies demonstrated the functional heterogeneity separating the ACC into cognitive (dorsal) and affective (rostral) subdivisions. ${ }^{77}$ In contrast to task-based approaches, Margulies et $a l^{78}$ used resting-state functional connectivity to examine ACC functional segregation. They replicated a rostral/caudal
ACC distinction, but also found a negative relationship (ie, anticorrelation) between the subgenual portions of the ACC and superior parietal regions, which are involved in higher order cognitive processes, such as attentional control and spatial WM. $^{79}$ Although the present study was not designed to disentangle task-related reciprocity between brain networks, we hypothesise that the observed additional sACC deactivation in the $\mathrm{PHx}$ group reflects the recruitment of additional neuronal resources (via anticorrelation) for successful task performance.

Within the larger context of the limbic-striato-pallidio-thalamic circuits implicated in the pathophysiology of primary depression, the functional implications of cortical-limbic circuits involving the medial prefrontal network merit comment in light of the abundant basic science literature available to guide translational models. ${ }^{80}$ Specifically, the ACC receives extensive dopaminergic innervation from the ventral tegmental area (VTA) and sends projections to the VTA that regulate phasic dopamine (DA) release. In rats, stimulation of the mPFC areas elicits burst firing patterns in the VTA-DA neurons while inactivation of the mPFC converts burst firing patterns to pacemaker-like firing activity. ${ }^{80}$ The burst firing patterns increase DA release in the nucleus accumbens, which is thought to encode information about reward prediction. ${ }^{81}$ The mesio-limbic DA projections from the VTA to the nucleus accumbens and the mPFC thus play major roles in learning associations between operant behaviours or sensory stimuli and reward. ${ }^{82}$ Further research is required to examine whether sACC dysfunction deleteriously affects the cortical drive on VTA-DA neuronal burst firing activity, which may impair reward learning in TLE patients with affective morbidity.

Previous research indicates that a lack of DMN deactivation, as found in association with psychiatric disturbance, is associated with impaired cognitive performance and deactivation in controls with performance gains. ${ }^{57}$ As predicted, we observed poorer WM performance in the $\mathrm{PHx}$ group that was related to increased deactivation, indicating that deactivation is not always cognitively advantageous. Whether this is a task-specific effect requires further investigation.

The behavioural performance of the two patient groups, relative to the control group, requires consideration. We found that both patient groups, irrespective of lifetime affective morbidity, performed less well at the 0 dot-back task condition compared 
with controls. We strongly suspect this is due to disease burden and the uncontrolled effects of antiepileptic medication on the attention networks. In the 1 dot-back condition, the PHx group performed more poorly than both control groups; whereas in the 2 dot-back condition, both TLE groups exhibited equal performance level. Collectively, these findings are suggestive of a 'weighted' interaction between psychopathology and TLE. Consistent with this hypothesis, previous research has reported that TLE patients per se are poorer on the 2 dot-back condition. $^{53}$

Our findings also challenge the 'default-mode interference hypothesis, ${ }^{83}$ which proposes that in a normal functioning system, the task-negative (DMN) components will be reduced during the transition from rest to goal-directed action, which is anticorrelated with activation in the task-positive network. Episodic attentional lapses and deficits in performance are considered to be associated with intruding DMN activity during task-specific processing. The performance impairments in the PHx group in this study cannot be explained by reduced deactivation of the DMN activity. Although the default mode interference hypothesis does not involve an inverted U-shape aspect as yet, ${ }^{83}$ it may be that reduced deactivation of the DMN may preclude adequate task positive activation or, alternatively, increased deactivation may paradoxically result in hypervigilance to irrelevant aspects of the task thereby causing distractions. The investigation of these possible explanations requires further research. Although the majority of functional magnetic resonance imaging (fMRI) studies in primary affective disorders focus on emotional processing, ${ }^{84}$ there is some evidence that psychiatric medication has a modulating effect on cognitive processing (see ${ }^{85}$ for review). We explored medication effects by removing patients from the analysis who were currently taking antidepressant/anxiolytic medication $(n=5)$. Despite the loss of statistical power, we replicated our principal finding that the PHx group significantly deactivated the same cluster (sACC) more than the $\mathrm{HV}$ and $\mathrm{nPHx}$ groups.

Studies reporting increased task-related DMN activation have been informed by patients with a current affective diagnosis. ${ }^{44-4657}$ Our inclusion of current mood state (BDI-FS and BAI) as covariates in our primary analysis and the removal of PHx patients with moderate to severe symptoms in post hoc analyses indicate that the variance in the negative BOLD signal (deactivation) in the sACC for the PHx group was independent of current mood state or severity. We interpret this finding as evidence of an enduring trait rather than state-like abnormality in TLE patients with a lifetime history of affective psychopathology.

There are several methodological strengths of our study that further support our primary finding. This study employed a clinical diagnosis of lifetime affective disorders. No single method is optimal to assess psychiatric functioning in TLE, ${ }^{14}$ but the merit of a psychiatric evaluation is that it not only considers transient mood states as reflected by self-report indices, but the full context of psychopathology, including medicationrelated and peri-ictal symptoms. ${ }^{16}$ Furthermore, as all patients were assessed by a neuropsychiatrist investigating the prevalence of lifetime and current psychiatric morbidity, we feel confident that our TLE-only group had no cases of undiagnosed psychopathology which is often overlooked in neurological consultations. $^{86}$

We employed a cognitive fMRI paradigm that produces robust progressive deactivations in relation to increasing task demands in HV and TLE patients. ${ }^{53}$ Moreover, we did not generate a subtraction contrast at the individual level. Instead, we used progressive deactivation contrasts at a second-level analysis, increasing the confidence that our main effect is driven by increased progressive deactivation as cognitive load increases, rather than a lack of activation between rest and task. ${ }^{40}$

We also performed correlational analyses to evaluate whether differences in task performance were related to sACC deactivation in the PHx group. No significant relationship or trend was observed for any condition, suggesting that sACC deactivation is pathological and related to lifetime affective psychiatric morbidity.

Our study does not address the issue of causality. The lack of significant difference in deactivation of the sACC between those with current and previous affective diagnoses and the TLE group without a psychiatric history most likely reflects the small sizes of the subgroups. Further studies with larger groups are needed to clarify this matter.

Several limitations of this study should be noted. We did not include individuals with primary affective disorders as a comparison group, which does limit the interpretation of the findings. However, we were principally interested in the effect of depression/anxiety in the context of TLE on DMN function, based on consistent evidence for reduced DMN deactivation in primary affective disorders. ${ }^{40} 41{ }^{43}$ Our TLE groups were heterogeneous although divergent MRI abnormalities were evenly distributed across the groups. We have not accounted for the influence of AEDs on the DMN and/or WM function. ${ }^{87}$ The majority of our patients were taking AED polytherapy in a variety of combinations. There were no significant differences in the number of AEDs between patient groups. This serves to limit the generalisability of the current study to pharmaco-resistant TLE patients. In addition, our fMRI results failed to achieve significance with correction for multiple comparisons. However, the cluster extent was $>20$ voxels, reducing the likelihood of false positives. ${ }^{88}$

The present study is the first to address DMN-related deactivation in TLE patients with a lifetime diagnosis of affective psychopathology. We observed significant deactivation of an 'affective network' node (sACC) in patients with a lifetime affective diagnosis with increasing task demands that conferred no cognitive benefit. Post hoc imaging analyses indicated that this finding was not associated with structural differences in the sACC. Our findings suggest the combination of an affective disorder and TLE has a unique pathophysiological basis supporting the growing body of research indicating that affective psychopathology in TLE has a neurobiological correlate ${ }^{89}$ and, in this context, the DMN performs differently compared with network activity in primary affective disorders (reduced deactivation). ${ }^{4-43}$ Prospective serial neuroimaging studies are needed to unravel the causal mechanisms underlying task-related DMN functional alterations and affective psychopathology in TLE.

Acknowledgements We are grateful to the radiographers at the Epilepsy Society Unit, Philippa Bartlett, Jane Burdett and Elaine Williams, who scanned the patients and especially to the patients for their kind participation.

Funding This work received funding from the Henry Smith Charity along with support by the Wellcome Trust (programme grant 083148), and the Medical Research Council (clinical research training fellowship G0802012 to GPW). The Epilepsy Society scanner was supported by the Wolfson Trust and Epilepsy Society. The work was undertaken at University College London Hospital and University College London who are supported by the National Institute for Health Research University College London Hospitals Biomedical Research Centre.

Competing interests RAP and JS had full access to all of the data in the study and take responsibility for the integrity of the data and the accuracy of the data analysis.

Patient consent Obtained.

Ethics approval UCL Hospitals NHS Foundation Trust Ethics Committee. 
Provenance and peer review Not commissioned; externally peer reviewed.

Open Access This is an Open Access article distributed in accordance with the Creative Commons Attribution Non Commercial (CC BY-NC 3.0) license, which permits others to distribute, remix, adapt, build upon this work non-commercially, and license their derivative works on different terms, provided the original work is properly cited and the use is non-commercial. See: http://creativecommons.org/ licenses/by-nc/3.0/

\section{REFERENCES}

1 Kanner AM. Can neurobiological pathogenic mechanisms of depression facilitate the development of seizure disorders? Lancet Neurol 2012;11:1093-102.

2 Rai D, Kerr MP, McManus S, et al. Epilepsy and psychiatric comorbidity: a nationally representative population-based study. Epilepsia 2012;53:1095-103.

3 Cleary RA, Baxendale SA, Thompson PJ, et al. Predicting and preventing psychopathology following temporal lobe epilepsy surgery. Epilepsy Behav 2013;26:322-34.

4 Boylan LS, Flint LA, Labovitz DL, et al. Depression but not seizure frequency predicts quality of life in treatment-resistant epilepsy. Neurology 2004;62:258-61.

5 Christensen J, Vestergaard M, Mortensen PB, et al. Epilepsy and risk of suicide: a population-based case-control study. Lancet Neurol 2007:6:693-8.

6 Kanner AM. Depression in epilepsy: a complex relation with unexpected consequences. Curr Opin Neurol 2008;21:190-4.

7 Forsgren L, Nyström L. An incident case-referent study of epileptic seizures in adults. Epilepsy Res 1990;6:66-81.

8 Nilsson FM, Kessing LV, Bolwig TG. On the increased risk of developing late-onset epilepsy for patients with major affective disorder. J Affect Disord 2003;76:39-48.

9 Hesdorffer DC, Hauser WA, Olafsson E, et al. Depression and suicide attempt as risk factors for incident unprovoked seizures. Ann Neurol 2006;59:35-41.

10 Hesdorffer DC, Ishihara L, Mynepalli L, et al. Epilepsy, suicidality, and psychiatric disorders: a bidirectional association. Ann Neurol 2012;72:184-91.

11 Adelöw C, Andersson T, Ahlbom A, et al. Hospitalization for psychiatric disorders before and after onset of unprovoked seizures/epilepsy. Neurology 2012;78:396-401.

12 Hitiris N, Mohanraj R, Norrie J, et al. Predictors of pharmacoresistant epilepsy. Epilepsy Res 2007;75:192-6.

13 Anhoury S, Brown RJ, Krishnamoorthy ES, et al. Psychiatric outcome after temporal lobectomy: a predictive study. Epilepsia 2000;41:1608-15.

14 Guarnieri R, Walz R, Hallak JE, et al. Do psychiatric comorbidities predict postoperative seizure outcome in temporal lobe epilepsy surgery? Epilepsy Behav 2009;14:529-34

15 Kanner AM, Byrne R, Chicharro A, et al. A lifetime psychiatric history predicts worse seizure outcome following temporal lobectomy. Neurology 2009;72:793-9.

16 Cleary RA, Thompson PJ, Fox Z, et al. Predictors of psychiatric and seizure outcome following temporal lobe epilepsy surgery. Epilepsia 2012;53:1705-12.

17 Drevets WC, Savitz J, Trimble M. The Subgenual Anterior Cingulate Cortex in Mood Disorders. CNS Spectr 2008;13:663-81.

18 Price JL, Drevets WC. Neurocircuitry of Mood Disorders. Neuropsychopharmacology 2010;35:192-216.

19 Tebartz van Elst L, Woermann FG, Lemieux L, et al. Amygdala enlargement in dysthymia--a volumetric study of patients with temporal lobe epilepsy. Biol Psychiatry 1999;461:1614-23.

20 Quiske A, Helmstaedter C, Lux S, et al. Depression in patients with temporal lobe epilepsy is related to mesial temporal sclerosis. Epilepsy Res 2000;39:121-5.

21 Baxendale SA, Thompson PJ, Duncan JS. Epilepsy \& depression: the effects of comorbidity on hippocampal volume--a pilot study. Seizure 2005; 14:435-8.

22 Richardson EJ, Griffith HR, Martin RC, et al. Structural and functional neuroimaging correlates of depression in temporal lobe epilepsy. Epilepsy Behav 2007;10:242-9.

23 Shamim S, Hasler G, Liew C, et al. Temporal lobe epilepsy, depression, and hippocampal volume. Epilepsia 2009;50:1067-71.

24 Wrench JM, Wilson SJ, Bladin PF, et al. Hippocampal volume and depression: insights from epilepsy surgery. J Neurol Neurosurg Psychiatry 2009;80:539-44.

25 Finegersh A, Avedissian C, Shamim S, et al. Bilateral hippocampal atrophy in temporal lobe epilepsy: effect of depressive symptoms and febrile seizures. Epilepsia 2011:52:689-97.

26 Butler T, Blackmon K, McDonald CR, et al. Cortical thickness abnormalities associated with depressive symptoms in temporal lobe epilepsy. Epilepsy Behav 2012;23:64-7.

27 Pope RA, Centeno M, Flugel D, et al. Neural correlates of de novo depression following left temporal lobe epilepsy surgery: A voxel based morphometry study of pre-surgical structural MRI. Epilepsy Res (in press) http://authors.elsevier.com/sd/ article/S0920121113003355

28 Bromfield EB, Altshuler L, Leiderman DB, et al. Cerebral metabolism and depression in patients with complex partial seizures. Arch Neurol 1992;49:617-23.

29 Schmitz EB, Moriarty J, Costa DC, et al. Psychiatric profiles and patterns of cerebral blood flow in focal epilepsy: interactions between depression, obsessionality, and perfusion related to the laterality of the epilepsy. I Neurol Neurosurg Psychiatry 1997;62:458-63.
30 Savic I, Lindström P, Gulyás B, et al. Limbic reductions of 5-HT1A receptor binding in human temporal lobe epilepsy. Neurology 2004;62:1343-51.

31 Giovacchini G, Toczek MT, Bonwetsch $\mathrm{R}$, et al. 5-HT 1A receptors are reduced in temporal lobe epilepsy after partial-volume correction. J Nucl Med 2005; 46:1128-35.

32 Salzberg M, Taher T, Davie M, et al. Depression in temporal lobe epilepsy surgery patients: an FDG-PET study. Epilepsia 2006;47:2125-30.

33 Gilliam FG, Maton BM, Martin RC, et al. Hippocampal 1H-MRSI correlates with severity of depression symptoms in temporal lobe epilepsy. Neurology 2007:68:364-8

34 Hasler G, Bonwetsch R, Giovacchini G, et al. 5-HT1A receptor binding in temporal lobe epilepsy patients with and without major depression. Biol Psychiatry 2007:62:1258-64.

35 Theodore WH, Hasler G, Giovacchini G, et al. Reduced hippocampal 5HT1A PET receptor binding and depression in temporal lobe epilepsy. Epilepsia 2007;48:1526-30.

36 Lothe A, Didelot A, Hammers A, et al. Comorbidity between temporal lobe epilepsy and depression: a [18F]MPPF PET study. Brain 2008;131:2765-82.

37 Martinez A, Finegersh A, Cannon DM, et al. The 5-HT1A receptor and 5-HT transporter in temporal lobe epilepsy. Neurology 2013;80:1465-71.

38 Raichle ME, MacLeod AM, Snyder AZ, et al. A default mode of brain function. Proc Natl Acad Sci U S A 2001:98:676-82.

39 Raichle ME, Snyder AZ. A default mode of brain function: a brief history of an evolving idea. Neuroimage 2007;37:1083-90.

40 Whitfield-Gabrieli S, Ford JM. Default mode network activity and connectivity in psychopathology. Annu Rev Clin Psychol 2012;8:49-76.

41 Buckner RL, Andrews-Hanna JR, Schacter DL. The brain's default network: anatomy, function, and relevance to disease. Ann N Y Acad Sci 2008:1124:1-38.

42 Broyd SJ, Demanuele $C$, Debener $S$, et al. Default-mode brain dysfunction in mental disorders: a systematic review. Neurosci Biobehav Rev 2009;33:279-96.

43 Anticevic A, Cole MW, Murray JD, et al. The role of default network deactivation in cognition and disease. Trends Cogn Sci 2012;16:584-92.

44 Sheline YI, Barch DM, Price JL, et al. The default mode network and self-referential processes in depression. Proc Natl Acad Sci U S A 2009;106:1942-7.

45 Grimm S, Boesiger $P$, Beck J, et al. Altered negative BOLD responses in the default-mode network during emotion processing in depressed subjects. Neuropsychopharmacology 2009;34:932-43.

46 Frodl T, Scheuerecker J, Albrecht J, et al. Neuronal correlates of emotional processing in patients with major depression. World J Biol Psychiatry 2009:10:202-8

47 Greicius MD, Flores BH, Menon V, et al. Resting-state functional connectivity in major depression: abnormally increased contributions from subgenual cingulate cortex and thalamus. Biol Psychiatry 2007;62:429-37.

48 Li B, Liu L, Friston KJ, et al. A Treatment-Resistant Default Mode Subnetwork in Major Depression. Biol Psychiatry 2013;74:48-54. http://dx.doi.org/10.1016/ j.biopsych.2012.11.007

49 Sylvester CM, Corbetta M, Raichle ME, et al. Functional network dysfunction in anxiety and anxiety disorders. Trends Neurosci 2012;35:527-35.

50 Zhang Z, Lu G, Zhong Y, et al. Altered spontaneous neuronal activity of the default-mode network in mesial temporal lobe epilepsy. Brain Res 2010;1323:152-60.

51 Voets NL, Beckmann CF, Cole DM, et al. Structural substrates for resting network disruption in temporal lobe epilepsy. Brain 2012;135:2350-7.

52 Frings L, Schulze-Bonhage A, Spreer J, et al. Remote effects of hippocampal damage on default network connectivity in the human brain. J Neurol 2009;256:2021-9.

53 Stretton J, Winston G, Sidhu M, et al. Neural correlates of working memory in Temporal Lobe Epilepsy--an fMRI study. Neuroimage 2012;60:1696-703.

54 Chen S, Wu X, Lui S, et al. Resting-state fMRI study of treatment-naive temporal lobe epilepsy patients with depressive symptoms. Neuroimage 2012;60:299-304.

55 Doucet GE, Skidmore C, Sharan AD, et al. Functional connectivity abnormalities vary by amygdala subdivision and are associated with psychiatric symptoms in unilateral temporal epilepsy. Brain Cogn 2013;82:171-82.

56 Kemmotsu N, Kucukboyaci NE, Cheng CE, et al. Alterations in functional connectivity between the hippocampus and prefrontal cortex as a correlate of depressive symptoms in temporal lobe epilepsy. Epilepsy Behav 2013;29:552-9.

57 Fernandez-Corcuera P, Salvador R, Monte GC, et al. Bipolar depressed patients show both failure to activate and failure to de-activate during performance of working memory task. J Affect Disord 2013;143:170-8.

58 Beck AT, Steer RA, Brown GK. Manual for the Beck Depression Inventory-FastScreen for Medical Patients. San Antonio, TX: Psychological Corporation, 2000.

59 Beck AT, Epstein N, Brown G, et al. An inventory for measuring clinical anxiety: psychometric properties. J Consult Clin Psychol 1988;56:893-7.

60 Cook PA, Symms M, Boulby PA, et al. Optimal acquisition orders of diffusion-weighted MRI measurements. J Magn Reson Imaging 2007;25:1051-8.

61 Callicott JH, Mattay VS, Bertolino A, et al. Physiological characteristics of capacity constraints in working memory as revealed by functional MRI. Cereb Cortex 1999:9:20-6 
62 Vollmar C, O'Muicheartaigh J, Barker GJ, et al. Motor system hyperconnectivity in juvenile myoclonic epilepsy: a cognitive functional magnetic resonance imaging study. Brain 2011;134:1710-19.

63 Jenkinson $M$, Bannister $P, B$ rady $M$, et al. Improved optimization for the robust and accurate linear registration and motion correction of brain images. Neuroimage 2002;17:825-41.

64 Behrens TE, Berg HJ, Jbabdi S, et al. Probalistic diffusion tractography with multiple fibre orientations: What can we gain? Neuroimage 2007:34:144-55.

65 Drevets WC, Price JL, Simpson JR Jr, et al. Subgenual prefrontal cortex abnormalities in mood disorders. Nature 1997;386:824-7.

66 Mayberg HS, Liotti M, Brannan SK, et al. Reciprocal limbic-cortical function and negative mood: converging PET findings in depression and normal sadness. Am J Psychiatry 1999;156:675-82

67 Mayberg HS, Lozano AM, Voon V, et al. Deep brain stimulation for treatment-resistant depression. Neuron 2005;45:651-60.

68 Danielson NB, Guo JN, Blumenfeld $H$. The default mode network and altered consciousness in epilepsy. Behav Neurol 2011:24:55-65.

69 Laufs H, Hamandi K, Salek-Haddadi A, et al. Temporal lobe interictal epileptic discharges affect cerebral activity in "default mode" brain regions. Hum Brain Mapp 2007;28:1023-32.

70 Zhang Z, Lu G, Zhong Y, et al. fMRI study of mesial temporal lobe epilepsy using amplitude of low-frequency fluctuation analysis. Hum Brain Mapp 2010; 31:1851-61.

71 Kobayashi E, Grova C, Tyvaert L, et al. Structures involved at the time of temporal lobe spikes revealed by interindividual group analysis of EEG/fMRI data. Epilepsia 2009:50:2549-56.

72 Petrovski S, Szoeke CE, Jones NC, et al. Neuropsychiatric symptomatology predicts seizure recurrence in newly treated patients. Neurology 2010;75:1015-21.

73 Anhoury S, Brown RJ, Kishnamoorthy ES, et al. Psychiatric outcome after temporal lobectomy: a predictive study. Epilepsia 2000;41:1608-15.

74 Guarnieri R, Walz R, Hallak JE, et al. Do psychiatric comorbidities predict postoperative outcome in temporal lobe epilepsy surgery. Epilepsy Behav 2009;14:529-34.

75 Kanner AM, Bryne R, Chicharro A, et al. A lifetime psychiatric history predicts a worse seizure outcome following temporal lobectomy. Neurology 2009;72:793-9.
76 Kleen JK, Scott RC, Holmes GL, et al. Hippocampal interictal epileptiform activity disrupts cognition in humans. Neurology 2013;81:18-24.

77 Bush G, Luu P, Posner MI. Cognitive and emotional influences in anterior cingulate cortex. Trends Cogn Sci 2000:4:215-22.

78 Margulies DS, Kelly AM, Uddin LQ, et al. Mapping the functional connectivity of anterior cingulate cortex. Neuroimage 2007;37:579-88.

79 Ikkai A, Curtis CE. Common neural mechanisms supporting spatia working memory, attention and motor intention. Neuropsychologia 2011:49:1428-34.

80 Price JL, Drevets WC. Neural circuits underlying the pathophysiology of mood disorders. Trends Cogn Sci 2012;16:61-71.

81 Treadway MT, Zald DH. Reconsidering anhedonia in depression: lessons from translational neuroscience. Neurosci Biobehav Rev 2011;35:537-55.

82 Dunlop BW, Numeroff CB. The role of dopamine in the pathophysiology of depression. Arch Gen Psychiatry 2007;64:327-37.

83 Sonuga-Barke EJ, Castellanos FX. Spontaneous attentional fluctuations in impaired states and pathological conditions: a neurobiological hypothesis. Neurosci Biobehav Rev 2007;31:977-86.

84 Delaveau $\mathrm{P}$, Jabourian $M$, Lemogne $C$, et al. Brain effects of antidepressants in major depression: a meta-analysis of emotional processing studies. J Affect Disord 2011;130:66-74

85 Bellani M, Dusi N, Yeh PH, et al. The effects of antidepressants on human brain as detected by imaging studies. Focus on major depression. Prog Neuropsychopharmacol Biol Psychiatry 2011;35:1544-52.

86 Kanner AM. Depression in epilepsy: prevalence, clinical semiology, pathogenic mechanisms, and treatment. Biol Psychiatry 2003;54:388-98.

87 Mula M, Trimble MR. Antiepileptic drug-induced cognitive adverse effects: potential mechanisms and contributing factors. CNS Drugs 2009:23:121-37.

88 Forman SD, Cohen JD, Fitzgerald M, et al. Improved assessment of significant activation in functional magnetic resonance imaging (fMRI): use of cluster-size threshold. Magn Reson Med 1995;33:636-47.

89 Gilliam FG, Santos J, Vahle V, et al. Depression in epilepsy: ignoring clinical expression of neuronal network dysfunction? Epilepsia 2004;45: 28-33. 